\title{
A condition for a closed one-form to be exact
}

\author{
Katsuhiro Moriya
}

\begin{abstract}
A condition for a closed one-form to be exact, the one-form having values in Euclidean space, on a compact surface without boundary, is given in the case where the surface has suitable differentiable automorphisms. Tori and hyperelliptic curves, with holomorphic automorphisms, are in this case. A local representation formula for surfaces in Euclidean space is then globalized. A condition for a local surface of constant mean curvature to be global, can be written using a harmonic Gauss map.
\end{abstract}

Mathematics Subject Classification (2010). Primary 58A10; Secondary $53 \mathrm{~A} 10$.

Keywords. Exact one-form, closed one-form, differentiable automorphism, period.

\section{Introduction}

The theory of de Rham cohomology is related to the theory of surfaces in Euclidean space. A surface is a smooth map $f$, from a smooth orientable connected two-dimensional manifold $M$ without boundary, to an $n$-dimensional Euclidean space $\mathbb{R}^{n}$. The differential $d f$ is an exact one-form on $M$ with values in $\mathbb{R}^{n}$. Hence, a surface represents a boundary of the first de Rham cohomology group of one-forms on $M$, with values in $\mathbb{R}^{n}$. The $f$ is reconstructed from its differential by the integral formula

$$
f(p)=\int_{\gamma} d f+f\left(p_{0}\right),
$$

with a curve $\gamma$ starting at $p_{0} \in M$ and ending at $p \in M$.

If $M$ is simply connected, then a one-form is exact if and only if the oneform is closed. In this case, there are several ways to construct differentials of surfaces. For example, the Weierstrass-Enneper representation formula for minimal surfaces in $\mathbb{R}^{3}$ constructs a differential of a minimal surface in $\mathbb{R}^{3}$,

This work was supported by Grant-in-Aid for Scientific Research(C) 22540064. The Ministry of Education, Culture, Sports, Science and Technology, Japan. 
from a meromorphic function and a holomorphic one-form on a Riemann surface (see Osserman [13]). The Kenmotsu formula constructs a differential of a surface in $\mathbb{R}^{3}$, from a non-zero mean curvature function and a Gauss map (see Kenmotsu [8]). These researches have been taken over by many researchers and developed in a variety of methods (see, for example, Hoffman and Osserman [6], Konopelchenko [12], Taimanov [18], [17], [16], [15], Friedrich [4], Pedit and Pinkall [14], and Burstall, Ferus, Leschke, Pedit and Pinkall [2]). Except [6], these researches are related with the spinorial or twistorial formulation of the theory of surfaces (see, for example, Bryant [1], Friedrich [5], Kamberov, Norman, Pedit, and Pinkall [7]).

In the case where the topology of $M$ is more complicated, these formulae do not construct an exact one-form in general, but a closed one-form. This motivates us to study a condition for a closed one-form to be exact.

We assume that $M$ is compact without boundary and of genus $g$. We embed $\mathbb{R}^{n}$ into the Clifford algebra $\mathrm{C} \ell_{n}$. We denote the first de Rham cohomology group of $\mathrm{C} \ell_{n}$-valued one-forms on $M$ by $\mathrm{Rh}^{1}$, and the cohomology class where a closed one-form $\eta$ belongs by $\llbracket \eta \rrbracket$. We define a pairing $(, \quad): \mathrm{Rh}^{1} \times \mathrm{Rh}^{1} \rightarrow \mathrm{C} \ell_{n}$ by

$$
(\llbracket \eta \rrbracket, \llbracket \xi \rrbracket):=\int_{M} \eta \wedge \xi .
$$

The dimension of $\mathrm{Rh}^{1}$ is $2 g$. Let $\delta_{1}, \ldots, \delta_{2 g}$ be a basis of $\mathrm{Rh}^{1}$. It is well-known that a closed one-form $\omega$ on $M$ is exact, if and only if

$$
\left(\llbracket \omega \rrbracket, \delta_{i}\right)=0
$$

for all $\delta_{1}, \ldots, \delta_{2 g} \in \mathrm{Rh}^{1}$. In general, it is difficult to find $\delta_{1}, \ldots, \delta_{g}$ and calculate the above $2 g$ integrals.

To ease this difficulty, we assume that $M$ has suitable differentiable automorphisms. We denote by $H_{1}(M, \mathbb{Z})$ the first homology group of $M$ with integer coefficients. For a closed curve $\gamma$ in $M$, we denote by $\llbracket \gamma \rrbracket$ the homology class where $\gamma$ belongs. Let $a_{1}, \ldots, a_{g}, b_{1}, \ldots, b_{g}$ be closed curves in $M$ such that $\llbracket a_{1} \rrbracket, \ldots, \llbracket a_{g} \rrbracket, \llbracket b_{1} \rrbracket, \ldots, \llbracket b_{g} \rrbracket$ is a canonical basis of $H_{1}(M, \mathbb{Z})$. We denote by $E_{g}$ the $g$ by $g$ unit matrix. Let $J_{2 g}$ be the $2 g$ by $2 g$ matrix defined by

$$
J_{2 g}:=\left(\begin{array}{cc}
O & E_{g} \\
-E_{g} & O
\end{array}\right) .
$$

We denote by $\operatorname{Sp}(g, \mathbb{Z})$ the symplectic group of $2 g$ by $2 g$ matrices with entries in $\mathbb{Z}$. For a matrix $N$, we denote its transpose by $N^{T}$. Then $\operatorname{Sp}(g, \mathbb{Z})=$ $\left\{X \mid X J_{2 g} X^{T}=J_{2 g}\right\}$. Let $\mathcal{A}$ be the group of differentiable automorphisms of $M$. A representation $h=\left(h_{j k}\right): \mathcal{A} \rightarrow \operatorname{Sp}(g, \mathbb{Z})$ is defined by the equation

$$
\begin{aligned}
& \left(\llbracket \mu\left(a_{1}\right) \rrbracket \quad \ldots \quad \llbracket \mu\left(a_{g}\right) \rrbracket \quad \llbracket \mu\left(b_{1}\right) \rrbracket \quad \ldots \quad \llbracket \mu\left(b_{g}\right) \rrbracket\right) \\
& =\left(\begin{array}{llllll}
\llbracket a_{1} \rrbracket & \ldots & \llbracket a_{g} \rrbracket & \llbracket b_{1} \rrbracket & \ldots & \left.\llbracket b_{g} \rrbracket\right) h(\mu) .
\end{array}\right.
\end{aligned}
$$

We decompose the matrix $J_{2 g} h^{T}-h J_{2 g}$ into a diagonal matrix $B(\mu)=$ $\left(b_{i j}(\mu)\right)$, and a matrix $C(\mu)=\left(c_{i j}(\mu)\right)$ such that the entries of the main diagonal are zero. Then, $J_{2 g} h^{T}(\mu)-h(\mu) J_{2 g}=B(\mu)+C(\mu)$. Let $\tilde{C}(\mu)=$ 
$\left(\tilde{c}_{i j}(\mu)\right)=\left(\left|c_{i j}(\mu)\right|\right)$ and $\Phi: C \ell_{n} \rightarrow \mathbb{R} 1$ be the projection. Then we have the following condition.

Theorem 1.1. We assume that there exist $\mu_{1}, \ldots, \mu_{m} \in \mathcal{A}$ and $r_{1}, \ldots, r_{m} \in$ $\mathbb{R} \backslash\{0\}$, satisfying one of the following conditions:

1. $\sum_{l=1}^{m}\left(r_{l} B\left(\mu_{l}\right)-\left|r_{l}\right| \tilde{C}\left(\mu_{l}\right)\right)$ is positive definite,

2. $\sum_{l=1}^{m}\left(r_{l} B\left(\mu_{l}\right)+\left|r_{l}\right| \tilde{C}\left(\mu_{l}\right)\right)$ is negative definite.

Let $\omega$ be a one-form on $M$ with

$$
\sum_{l=1}^{m} r_{l}\left(\omega \wedge \mu_{l}{ }^{*} \omega-\mu_{l}{ }^{*} \omega \wedge \omega\right) \neq 0
$$

$A$ one-form $\omega$ with values in $\mathbb{R}^{n}$ is exact, if and only if $\omega$ is closed and

$$
\Phi\left(\sum_{l=1}^{m} r_{l}\left[\left(\llbracket \omega \rrbracket, \llbracket \mu_{l}^{*} \omega \rrbracket\right)-\left(\llbracket \mu_{l}^{*} \omega \rrbracket, \llbracket \omega \rrbracket\right)\right]\right)=0 .
$$

If $\sum_{l=1}^{m} r_{l}\left(\omega \wedge \mu_{l}{ }^{*} \omega-\mu_{l}{ }^{*} \omega \wedge \omega\right)$ is a non-zero exact one-form, then we understand that $\omega$ is an exact one-form, without integration. We see examples in the case where $M$ is a square torus (Corollary 4.1), a hexagonal torus (Corollary 4.2), and a hyperelliptic curve with affine plane model

$$
\left\{(z, w) \in \mathbb{C} \times \mathbb{C} \mid w^{2}=z^{2(g+1)}-1\right\}
$$

(Corollary 5.1).

We identify $\mathbb{R}^{4}$ with quaternions $\mathbb{H}$. For $a \in \mathbb{H}$, we denote its conjugate by $\bar{a}$. Then we have the following similar condition.

Theorem 1.2. We assume that there exist $\mu_{1}, \ldots, \mu_{m} \in \mathcal{A}$ and $r_{1}, \ldots, r_{m} \in$ $\mathbb{R} \backslash\{0\}$, satisfying one of the following conditions:

1. $\sum_{l=1}^{m}\left(r_{l} B\left(\mu_{l}\right)-\left|r_{l}\right| \tilde{C}\left(\mu_{l}\right)\right)$ is positive definite,

2. $\sum_{l=1}^{m}\left(r_{l} B\left(\mu_{l}\right)+\left|r_{l}\right| \tilde{C}\left(\mu_{l}\right)\right)$ is negative definite.

Let $\omega$ be a one-form on $M$ with

$$
\sum_{l=1}^{m} r_{l}\left(\omega \wedge \mu_{l}{ }^{*} \bar{\omega}-\mu_{l}{ }^{*} \omega \wedge \bar{\omega}\right) \neq 0
$$

$A$ one-form $\omega$ with values in $\mathbb{H}$ is exact, if and only if $\omega$ is closed and

$$
\sum_{l=1}^{m} r_{l}\left[\left(\llbracket \omega \rrbracket, \llbracket \mu_{l}^{*} \bar{\omega} \rrbracket\right)-\left(\llbracket \mu_{l}^{*} \omega \rrbracket, \llbracket \bar{\omega} \rrbracket\right)\right]=0
$$

By Theorem 1.2, we have a property of a period of a one-form. We denote the inner product of $\mathbb{R}^{4}$ by $\langle$,$\rangle . Let \eta$ be a one-form with values in $\operatorname{Im} \mathbb{H}$, such that

$$
\int_{a_{1}} \eta \neq 0, \quad \int_{a_{2}} \eta=\cdots=\int_{a_{g}} \eta=\int_{b_{1}} \eta=\cdots=\int_{b_{g}} \eta=0 .
$$

We can consider $\eta$ as a differential of a singly-periodic surface in $\mathbb{R}^{3}$. 
Corollary 1.3. We assume that there exist $\mu \in \mathcal{A}$ and $r \in \mathbb{R} \backslash\{0\}$, satisfying one of the following conditions:

1. $r B(\mu)-|r| \tilde{C}(\mu)$ is positive definite,

2. $r B(\mu)+|r| \tilde{C}(\mu)$ is negative definite.

Let $\eta$ be an $\operatorname{Im} \mathbb{H}$-valued one-form such that

$$
\int_{a_{1}} \eta \neq 0, \quad \int_{a_{2}} \eta=\cdots=\int_{a_{g}} \eta=\int_{b_{1}} \eta=\cdots=\int_{b_{g}} \eta=0 .
$$

We assume that

$$
r\left(\eta \wedge \mu^{*} \bar{\eta}-\mu^{*} \eta \wedge \bar{\eta}\right) \neq 0
$$

Then

$$
\left\langle\int_{a_{1}} \eta, \int_{b_{1}} \mu^{*} \eta\right\rangle \neq 0 .
$$

Returning the initial motivation, we combine Theorem 1.2 and the construction of a closed one-form for a surface in $\mathbb{R}^{4}$ in [2]. We denote the complex structure of $M$ by $J$. For a one-form $\omega$ on $M$, we define $* \omega:=\omega \circ J$. We denote the set of real parts of quaternions by Re $\mathbb{H}$ and the set of imaginary parts of quaternions by $\operatorname{Im} \mathbb{H}$. If $f$ is an immersion, then there exists a map $N: M \rightarrow S^{2} \subset \operatorname{Im} \mathbb{H}$ such that $* d f=N d f$. We call a non-constant map $f: M \rightarrow \mathbb{R}^{4}$ a surface if there exists $N: M \rightarrow S^{2} \subset \operatorname{Im} \mathbb{H}$ such that $* d f=N d f$. Then we have the following condition.

Corollary 1.4. We assume that there exist $\mu_{1}, \ldots, \mu_{m} \in \mathcal{A}$ and $r_{1}, \ldots, r_{m} \in$ $\mathbb{R} \backslash\{0\}$, satisfying one of the following conditions:

1. $\sum_{l=1}^{m}\left(r_{l} B\left(\mu_{l}\right)-\left|r_{l}\right| \tilde{C}\left(\mu_{l}\right)\right)$ is positive definite,

2. $\sum_{l=1}^{m}\left(r_{l} B\left(\mu_{l}\right)+\left|r_{l}\right| \tilde{C}\left(\mu_{l}\right)\right)$ is negative definite.

We assume that maps $N: M \rightarrow S^{2} \subset \operatorname{Im} \mathbb{H}$ and $\mathcal{H}: M \rightarrow \mathbb{H} \backslash\{0\}$, and a non-zero one-form $\omega$ on $M$ satisfy

$$
\begin{gathered}
-2 \omega \overline{\mathcal{H}}=* d N+N d N, 2 \omega \wedge d \overline{\mathcal{H}}=d * d N+d N \wedge d N, \\
\sum_{l=1}^{m} r_{l}\left(\omega \wedge \mu_{l}{ }^{*} \bar{\omega}-\mu_{l}{ }^{*} \omega \wedge \bar{\omega}\right) \neq 0, \\
\sum_{l=1}^{m} r_{l}\left[\left(\llbracket \omega \rrbracket, \llbracket \mu_{l}{ }^{*} \bar{\omega} \rrbracket\right)-\left(\llbracket \mu_{l}{ }^{*} \omega \rrbracket, \llbracket \bar{\omega} \rrbracket\right)\right]=0 .
\end{gathered}
$$

Then there exists a surface $f: M \rightarrow \mathbb{R}^{4}$ with mean curvature vector field $\mathcal{H}$, such that $d f=\omega$ and $* d f=N d f$.

If $f$ takes values in $\operatorname{Im} \mathbb{H} \cong \mathbb{R}^{3}$, then $* d f=N d f=-d f N$. Applying Corollary 1.2 to surfaces of constant mean curvature, we have the following condition.

Corollary 1.5. We assume that there exist $\mu_{1}, \ldots, \mu_{m} \in \mathcal{A}$ and $r_{1}, \ldots, r_{m} \in$ $\mathbb{R} \backslash\{0\}$, satisfying one of the following conditions: 
1. $\sum_{l=1}^{m}\left(r_{l} B\left(\mu_{l}\right)-\left|r_{l}\right| \tilde{C}\left(\mu_{l}\right)\right)$ is positive definite,
2. $\sum_{l=1}^{m}\left(r_{l} B\left(\mu_{l}\right)+\left|r_{l}\right| \tilde{C}\left(\mu_{l}\right)\right)$ is negative definite.

We assume that a non-conformal harmonic map $N: M \rightarrow S^{2} \subset \operatorname{Im} \mathbb{H}$

$$
\begin{gathered}
\sum_{l=1}^{m} r_{l}\left(N * d N \wedge \mu_{l}^{*}(N * d N)-\mu_{l}^{*}(N * d N) \wedge(N * d N)\right) \neq 0, \\
\sum_{l=1}^{m} r_{l}\left[\left(\llbracket N * d N \rrbracket, \llbracket \mu_{l}^{*}(N * d N) \rrbracket\right)-\left(\llbracket \mu_{l}^{*}(N * d N) \rrbracket, \llbracket N * d N \rrbracket\right)\right]=0 .
\end{gathered}
$$

Then there exists a surface $f: M \rightarrow \operatorname{Im} \mathbb{H} \cong \mathbb{R}^{3}$ of non-zero constant mean curvature, such that $* d f=N d f=-d f N$.

\section{Clifford algebra-valued one-forms}

Throughout this paper, we assume that all manifolds, maps, and differential forms are smooth. In this section, we show an analog of a relation between the periods of two complex-valued, closed one-forms (see Farkas and Kra [3], III.2.3. Proposition). Then we prove Theorem 1.1.

Let $\left\{e_{1}, \ldots, e_{n}\right\}$ be an orthonormal basis of $\mathbb{R}^{n}$. The Clifford algebra $\mathrm{C} \ell_{n}$ is the algebra generated by $e_{1}, \ldots, e_{n}$ subject to the relation

$$
e_{i} e_{j}+e_{j} e_{i}=-2 \delta_{i j}
$$

We denote the projection $\mathrm{C}_{n} \rightarrow \mathbb{R}^{1}$ by $\Phi$. Let $\langle u, v\rangle$ be the inner product of $u$ and $v \in \mathbb{R}^{n}$ and $|u|:=\langle u, u\rangle^{1 / 2}$ the norm of $\mathbb{R}^{n}$. Let $u:=\sum_{i=1}^{n} u_{i} e_{i}$, $v:=\sum_{i=1}^{n} v_{i} e_{i} \in \mathbb{R}^{n} \subset \mathrm{C} \ell_{n}\left(u_{1}, \ldots, u_{n}, v_{1}, \ldots, v_{n} \in \mathbb{R}\right)$. Then

$$
u v=-\langle u, v\rangle+\sum_{i<j}\left|\begin{array}{ll}
u_{i} & u_{j} \\
v_{i} & v_{j}
\end{array}\right| e_{i} e_{j}
$$

We identify the Clifford algebra and the exterior algebra in a natural manner. Then $u v=-\langle u, v\rangle+u \wedge v$. Hence $u$ and $v$ are linearly independent over $\mathbb{R}$, if and only if $u v-v u \neq 0$. Let $\alpha: \mathrm{C}_{n} \rightarrow \mathrm{C} \ell_{n}$ be the automorphism which extends $\alpha(u)=-u$ for $u \in \mathbb{R}^{n}$. The map $\delta: C \ell_{2} \rightarrow \mathbb{H}$, which extends

$$
\delta(1)=1, \quad \delta\left(e_{1}\right)=i, \quad \delta\left(e_{2}\right)=j, \quad \delta\left(e_{1} e_{2}\right)=k,
$$

is an isomorphism between $\mathrm{C}_{2}$ and $\mathbb{H}$.

Let $M$ be a compact oriented two-dimensional manifold without boundary. We assume that the genus of $M$ is $g$. For closed curves $\gamma_{1}$ and $\gamma_{2}$ in $M$, we denote $\gamma_{1} \cdot \gamma_{2}$ the intersection number of $\gamma_{1}$ and $\gamma_{2}$. Let $\pi_{1}(M)=\pi_{1}\left(M, p_{0}\right)$ be the fundamental group of $M$ with base point $p_{0} \in M$. For a closed curve $u$ with initial and end point $p_{0}$ in $M$, we denote the inverse curve of $u$ by $u^{-1}$, and the homotopy class that $u$ represents by $[u]$. The map from $\pi_{1}(M)$ to $H_{1}(M, \mathbb{Z})$ defined by $[u] \mapsto \llbracket u \rrbracket$ is a surjective group homomorphism. We fix simple closed curves

$$
\left\{a_{1}, \ldots, a_{g}, b_{1}, \ldots, b_{g}\right\}
$$


in $M$ with initial and end point $p_{0} \in M$, such that all curves are disjoint from each other, except $p_{0}$, that

$$
a_{i} \cdot a_{j}=b_{i} \cdot b_{j}=0, a_{i} \cdot b_{j}=-b_{j} \cdot a_{i}=\delta_{i j}(i, j=1, \ldots, g),
$$

and that

$$
\left[a_{1} b_{1} a_{1}^{-1} b_{1}^{-1} \cdots a_{g} b_{g} a_{g}^{-1} b_{g}^{-1}\right]=1
$$

The ordered cycles

$$
\llbracket a_{1} \rrbracket, \ldots, \llbracket a_{g} \rrbracket, \llbracket b_{1} \rrbracket, \ldots, \llbracket b_{g} \rrbracket
$$

form a canonical basis of $H_{1}(M, \mathbb{Z})$. We assume that $\llbracket \gamma \rrbracket \in H_{1}(M, \mathbb{Z})$ and $\llbracket \eta \rrbracket \in \mathrm{Rh}^{1}$. Then we can define a map $V_{\llbracket \gamma \rrbracket}: \mathrm{Rh}^{1} \rightarrow \mathrm{C} \ell_{n}$ by

$$
V_{\llbracket \gamma \rrbracket}(\llbracket \eta \rrbracket):=\int_{\gamma} \eta \text {. }
$$

We set

$$
\begin{aligned}
& P(\eta)=\left(\begin{array}{llllll}
P_{1}(\eta) & \ldots & P_{g}(\eta) & P_{g+1}(\eta) & \ldots & P_{2 g}(\eta)
\end{array}\right) \\
& :=\left(V_{\llbracket a_{1} \rrbracket}(\llbracket \eta \rrbracket) \quad \ldots \quad V_{\llbracket a_{g} \rrbracket}(\llbracket \eta \rrbracket) \quad V_{\llbracket b_{1} \rrbracket}(\llbracket \eta \rrbracket) \quad \ldots \quad V_{\llbracket b_{g} \rrbracket}(\llbracket \eta \rrbracket)\right) .
\end{aligned}
$$

Lemma 2.1. Let $\eta$ and $\xi$ be closed one-forms on $M$ with values in $\mathrm{C}_{n}$. Then

$$
(\llbracket \eta \rrbracket, \llbracket \xi \rrbracket)=P(\eta) J_{2 g} P(\xi)^{T}
$$

Proof. Let $\psi: U \rightarrow M$ be the universal covering. Then there exists a simply connected set $\tilde{M}$ with boundary $\partial \tilde{M}$ in $U$ such that

$$
\begin{gathered}
\partial \tilde{M}=\tilde{a}_{1} \tilde{b}_{1} \tilde{a}_{1}^{-} \tilde{b}_{1}^{-} \ldots \tilde{a}_{g} \tilde{b}_{g} \tilde{a}_{g}^{-} \tilde{b}_{g}^{-}, \\
\psi\left(\tilde{a}_{i}\right)=a_{i}, \quad \psi\left(\tilde{a}_{i}^{-}\right)=a_{i}^{-1}, \quad \psi\left(\tilde{b}_{i}\right)=b_{i}, \quad \psi\left(\tilde{b}_{i}^{-}\right)=b_{i}^{-1} \quad(i=1, \ldots, g) .
\end{gathered}
$$

(see Figure 1).

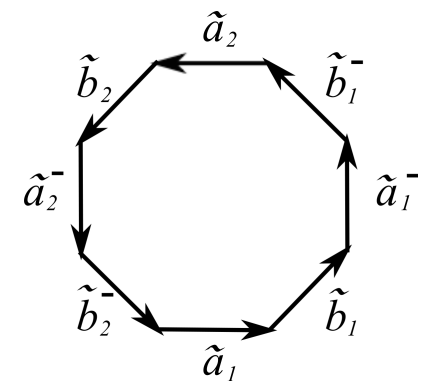

Figure 1. $\tilde{M}$ in the case where $g=2$.

We fix a point $z_{0} \in \tilde{M}$ such that $\psi\left(z_{0}\right)=p_{0}$. We denote a curve with initial point $z_{0}$ and end point $z$ in $\tilde{M}$ by $\gamma$. We can define a map $f: \tilde{M} \rightarrow \mathrm{C} \ell_{n}$ by

$$
f(z):=\int_{\gamma} \psi^{*} \eta .
$$


By Stokes' theorem, we have

$$
\begin{gathered}
(\llbracket \eta \rrbracket, \llbracket \xi \rrbracket)=\int_{M} \eta \wedge \xi=\int_{\tilde{M}} \psi^{*}(\eta \wedge \xi)=\int_{\tilde{M}} d f \wedge \psi^{*} \xi=\int_{\partial \tilde{M}} f \psi^{*} \xi \\
=\sum_{m=1}^{g} \int_{a_{m}+b_{m}+a_{m}^{-}+b_{m}^{-}} f \psi^{*} \xi
\end{gathered}
$$

For a point $z \in \tilde{a}_{m}$, we define $z^{\prime} \in \tilde{a}_{m}^{-}$by $\psi(z)=\psi\left(z^{\prime}\right)$. Let $\tilde{\gamma}$ be a curve in $\tilde{M}$ with initial point $z_{0}$ and end point $z^{\prime}$. Then

$$
\begin{gathered}
\int_{\tilde{a}_{m}+\tilde{a}_{m}^{-}} f \psi^{*} \xi=\int_{\tilde{a}_{m}}\left(\int_{\gamma} \psi^{*} \eta-\int_{\tilde{\gamma}} \psi^{*} \eta\right) \psi^{*} \xi \\
=-\int_{\tilde{b}_{m}} \psi^{*} \eta \int_{\tilde{a}_{m}} \psi^{*} \xi=-\int_{b_{m}} \eta \int_{a_{m}} \xi=-P_{g+m}(\eta) P_{m}(\xi) .
\end{gathered}
$$

Similarly, for a point $z \in \tilde{b}_{m}$, we define $z^{\prime} \in \tilde{b}_{m}^{-}$by $\psi(z)=\psi\left(z^{\prime}\right)$. Then

$$
\begin{gathered}
\int_{\tilde{b}_{m}+\tilde{b}_{m}^{-}} f \psi^{*} \xi=\int_{\tilde{b}_{m}}\left(\int_{\gamma} \psi^{*} \eta-\int_{\tilde{\gamma}} \psi^{*} \eta\right) \psi^{*} \xi \\
=\int_{\tilde{a}_{m}} \psi^{*} \eta \int_{\tilde{b}_{m}} \psi^{*} \xi=\int_{a_{m}} \eta \int_{b_{m}} \xi=P_{m}(\eta) P_{g+m}(\xi) .
\end{gathered}
$$

By (2.2), we have (2.1).

Proof of Theorem 1.1. For a closed curve $\gamma$ in $M$, we have

$$
V_{\llbracket \gamma \rrbracket}\left(\llbracket \tau^{*} \omega \rrbracket\right)=\int_{\gamma} \tau^{*} \omega=\int_{\tau(\gamma)} \omega=V_{\llbracket \tau(\gamma) \rrbracket}(\llbracket \omega \rrbracket) .
$$

Hence

$$
P\left(\mu_{l}^{*} \omega\right)=P(\omega) h\left(\mu_{l}\right)
$$

Then

$$
\left(\llbracket \omega \rrbracket, \llbracket \mu_{l}{ }^{*} \omega \rrbracket\right)=P(\omega) J_{2 g} P\left(\mu_{l}{ }^{*} \omega\right)^{T}=P(\omega) J_{2 g} h\left(\mu_{l}\right)^{T} P(\omega)^{T} .
$$

We have

$$
\left(P(\omega) J_{2 g} h\left(\mu_{l}\right)^{T} P(\omega)^{T}\right)^{T}=P(\omega) h\left(\mu_{l}\right) J_{2 g}^{T} P(\omega)=-\left(\llbracket \mu_{l}^{*} \omega \rrbracket, \llbracket \omega \rrbracket\right) .
$$

Let $\tilde{P}(\omega)=\left(\left|P_{1}(\omega)\right| \quad \ldots \quad\left|P_{2 g}(\omega)\right|\right)$.

We assume that $\sum_{l=1}^{m}\left(r_{l} B\left(\mu_{l}\right)-\left|r_{l}\right| \tilde{C}\left(\mu_{l}\right)\right)$ is positive definite. Then

$$
\begin{gathered}
0=\Phi\left(\sum_{l=1}^{m} r_{l}\left[\left(\llbracket \omega \rrbracket, \llbracket \mu_{l}^{*} \omega \rrbracket\right)-\left(\llbracket \mu_{l}^{*} \omega \rrbracket, \llbracket \omega \rrbracket\right)\right]\right) \\
=\Phi\left(P(\omega) \sum_{l=1}^{m} r_{l}\left[J_{2 g} h\left(\mu_{l}\right)^{T}-h\left(\mu_{l}\right) J_{2 g}\right] P(\omega)^{T}\right) \\
=\Phi\left(P(\omega) \sum_{l=1}^{m} r_{l}\left[B\left(\mu_{l}\right)+C\left(\mu_{l}\right)\right] P(\omega)^{T}\right)
\end{gathered}
$$




$$
\begin{gathered}
=-\sum_{l=1}^{m}\left(\sum_{i=1}^{2 g} r_{l} b_{i i}\left(\mu_{l}\right)\left|P_{i}(\omega)\right|^{2}+\sum_{i, j=1}^{2 g} r_{l} c_{i j}\left(\mu_{l}\right)\left\langle P_{i}(\omega), P_{j}(\omega)\right\rangle\right) \\
\leq-\sum_{l=1}^{m}\left(\sum_{i=1}^{2 g} r_{l} b_{i i}\left(\mu_{l}\right)\left|P_{i}(\omega)\right|^{2}-\sum_{i, j=1}^{2 g}\left|r_{l}\right|\left|c_{i j}\left(\mu_{l}\right)\right|\left|P_{i}(\omega)\right|\left|P_{j}(\omega)\right|\right) \\
=-\tilde{P}(\omega)\left(\sum_{l=1}^{m}\left(r_{l} B\left(\mu_{l}\right)-\left|r_{l}\right| \tilde{C}\left(\mu_{l}\right)\right)\right) \tilde{P}(\omega)^{T} \leq 0 .
\end{gathered}
$$

Hence $\tilde{P}(\omega)=0$.

We assume that $\sum_{l=1}^{m}\left(r_{l} B\left(\mu_{l}\right)+\left|r_{l}\right| \tilde{C}\left(\mu_{l}\right)\right)$ is negative definite. Then

$$
\begin{gathered}
0=\Phi\left(\sum_{l=1}^{m} r_{l}\left[\left(\llbracket \omega \rrbracket, \llbracket \mu_{l}^{*} \omega \rrbracket\right)-\left(\llbracket \mu_{l}{ }^{*} \omega \rrbracket, \llbracket \omega \rrbracket\right)\right]\right) \\
=-\sum_{l=1}^{m}\left(\sum_{i=1}^{2 g} r_{l} b_{i i}\left(\mu_{l}\right)\left|P_{i}(\omega)\right|^{2}+\sum_{i, j=1}^{2 g} r_{l} c_{i j}\left(\mu_{l}\right)\left\langle P_{i}(\omega), P_{j}(\omega)\right\rangle\right) \\
\geq-\sum_{l=1}^{m}\left(\sum_{i=1}^{2 g} r_{l} b_{i i}\left(\mu_{l}\right)\left|P_{i}(\omega)\right|^{2}+\sum_{i, j=1}^{2 g}\left|r_{l}\right|\left|c_{i j}\left(\mu_{l}\right)\right|\left|P_{i}(\omega)\right|\left|P_{j}(\omega)\right|\right) \\
=-\tilde{P}(\omega)\left(\sum_{l=1}^{m}\left(r_{l} B\left(\mu_{l}\right)+\left|r_{l}\right| \tilde{C}\left(\mu_{l}\right)\right)\right) \tilde{P}(\omega)^{T} \geq 0 .
\end{gathered}
$$

Hence $\tilde{P}(\omega)=0$.

A one-form $\omega$ is exact if and only if $\tilde{P}(\omega)=0$. Hence, Theorem 1.1 holds.

\section{Quaternionic-valued one-forms}

We have a similar condition for quaternionic-valued one-forms.

Proof of Theorem 1.2. We have

$$
\begin{gathered}
\left(\llbracket \omega \rrbracket, \llbracket \mu_{l}{ }^{*} \bar{\omega} \rrbracket\right)=P(\omega) J_{2 g} P\left(\mu_{l}{ }^{*} \bar{\omega}\right)^{T}=P(\omega) J_{2 g} h\left(\mu_{l}\right)^{T} P(\bar{\omega})^{T}, \\
\overline{\left(\llbracket \omega \rrbracket, \llbracket \mu_{l}{ }^{*} \bar{\omega} \rrbracket\right)}=\overline{\left(P(\omega) J_{2 g} P\left(\mu_{l}{ }^{*} \bar{\omega}\right)^{T}\right)^{T}} \\
=-P(\omega) h\left(\mu_{l}\right) J_{2 g} P(\bar{\omega})^{T}=-\left(\llbracket \mu_{l}{ }^{*} \omega \rrbracket, \llbracket \bar{\omega} \rrbracket\right) .
\end{gathered}
$$

Then

$$
\begin{gathered}
2 \operatorname{Re}\left(\llbracket \omega \rrbracket, \llbracket \mu_{l}{ }^{*} \bar{\omega} \rrbracket\right)=\left(\llbracket \omega \rrbracket, \llbracket \mu_{l}{ }^{*} \bar{\omega} \rrbracket\right)+\overline{\left(\llbracket \omega \rrbracket, \llbracket \mu_{l}{ }^{*} \bar{\omega} \rrbracket\right)} \\
=P(\omega) J_{2 g} h\left(\mu_{l}\right)^{T} P(\bar{\omega})^{T}-P(\omega) h\left(\mu_{l}\right) J_{2 g} P(\bar{\omega})^{T} \\
=P(\omega)\left(J_{2 g} h\left(\mu_{l}\right)^{T}-h\left(\mu_{l}\right) J_{2 g}\right) P(\bar{\omega})^{T} \\
=P(\omega)\left(B\left(\mu_{l}\right)+C\left(\mu_{l}\right)\right) P(\bar{\omega})^{T} .
\end{gathered}
$$


We assume that $\sum_{l=1}^{m}\left(r_{l} B\left(\mu_{l}\right)-\left|r_{l}\right| \tilde{C}\left(\mu_{l}\right)\right)$ is positive definite. Since $\langle u, v\rangle=$ $(u \bar{v}+\bar{v} u) / 2$ for $u, v \in \mathbb{H}$, we have

$$
\begin{gathered}
0=\sum_{l=1}^{m} r_{l}\left[\left(\llbracket \omega \rrbracket, \llbracket \mu_{l}^{*} \bar{\omega} \rrbracket\right)-\left(\llbracket \mu_{l}^{*} \omega \rrbracket, \llbracket \bar{\omega} \rrbracket\right)\right] \\
=P(\omega) \sum_{l=1}^{m} r_{l}\left[B\left(\mu_{l}\right)+C\left(\mu_{l}\right)\right] P(\bar{\omega})^{T} \\
=-\sum_{l=1}^{m}\left(\sum_{i=1}^{2 g} r_{l} b_{i i}\left(\mu_{l}\right)\left|P_{i}(\omega)\right|^{2}+\sum_{i, j=1}^{2 g} r_{l} c_{i j}\left(\mu_{l}\right)\left\langle P_{i}(\omega), P_{j}(\omega)\right\rangle\right) \\
\leq-\sum_{l=1}^{m}\left(\sum_{i=1}^{2 g} r_{l} b_{i i}\left(\mu_{l}\right)\left|P_{i}(\omega)\right|^{2}-\sum_{i, j=1}^{2 g}\left|r_{l}\right|\left|c_{i j}\left(\mu_{l}\right)\right|\left|P_{i}(\omega)\right|\left|P_{j}(\omega)\right|\right) \\
=-\tilde{P}(\omega)\left(\sum_{l=1}^{m}\left(r_{l} B\left(\mu_{l}\right)-\left|r_{l}\right| \tilde{C}\left(\mu_{l}\right)\right)\right) \tilde{P}(\bar{\omega})^{T} \leq 0 .
\end{gathered}
$$

Hence $\tilde{P}(\omega)=0$.

We assume that $\sum_{l=1}^{m}\left(r_{l} B\left(\mu_{l}\right)+\left|r_{l}\right| \tilde{C}\left(\mu_{l}\right)\right)$ is negative definite. Then

$$
\begin{gathered}
0=\sum_{l=1}^{m} r_{l}\left[\left(\llbracket \omega \rrbracket, \llbracket \mu_{l}{ }^{*} \bar{\omega} \rrbracket\right)-\left(\llbracket \mu_{l}{ }^{*} \omega \rrbracket, \llbracket \bar{\omega} \rrbracket\right)\right] \\
=-\sum_{l=1}^{m}\left(\sum_{i=1}^{2 g} r_{l} b_{i j}\left(\mu_{l}\right)\left|P_{i}(\omega)\right|^{2}+\sum_{i, j=1}^{2 g} r_{l} c_{i j}\left(\mu_{l}\right)\left\langle P_{i}(\omega), P_{j}(\omega)\right\rangle\right) \\
\geq-\sum_{l=1}^{m}\left(\sum_{i=1}^{2 g} r_{l} b_{i j}\left(\mu_{l}\right)\left|P_{i}(\omega)\right|^{2}+\sum_{i, j=1}^{2 g}\left|r_{l}\right|\left|c_{i j}\left(\mu_{l}\right)\right|\left|P_{i}(\omega)\right|\left|P_{j}(\omega)\right|\right) \\
=-\tilde{P}(\omega)\left(\sum_{l=1}^{m}\left(r_{l} B\left(\mu_{l}\right)+\left|r_{l}\right| \tilde{C}\left(\mu_{l}\right)\right)\right) \tilde{P}(\bar{\omega})^{T} \geq 0 .
\end{gathered}
$$

Hence $\tilde{P}(\omega)=0$.

A one-form $\omega$ is exact if and only if $\tilde{P}(\omega)=0$. Hence, Theorem 1.2 holds.

Proof of Corollary 1.3. Since $\eta$ is not exact, we have

$$
\int_{M} r\left(\eta \wedge \mu^{*} \bar{\eta}-\mu^{*} \eta \wedge \bar{\eta}\right) \neq 0
$$

On the other hand,

$$
\int_{M} r\left(\eta \wedge \mu^{*} \bar{\eta}-\mu^{*} \eta \wedge \bar{\eta}\right)=-r\left(\int_{a_{1}} \eta \int_{b_{1}} \mu^{*} \eta+\int_{b_{1}} \mu^{*} \eta \int_{a_{1}} \eta\right)
$$




$$
=-2 r\left\langle\int_{a_{1}} \eta, \int_{b_{1}} \mu^{*} \eta\right\rangle
$$

by Lemma 2.1. Hence Corollary 1.3 holds.

\section{One-forms on tori}

We review the classification of tori and their holomorphic automorphisms, and consider Theorem 1.1 and Theorem 1.2 in the case where $M$ is a torus.

Let $M$ be a torus. We consider $M$ as a Riemann surface. Then $M$ is biholomorphic to an orbit space $\mathbb{C} / \Lambda_{\lambda}$ with a lattice

$$
\Lambda_{\lambda}:=\mathbb{Z}+\mathbb{Z} \lambda, \quad \operatorname{Im} \lambda>0, \quad-\frac{1}{2}<\operatorname{Re} \lambda \leq \frac{1}{2}, \begin{cases}|\lambda| \geq 1 \quad & (\operatorname{Re} \lambda \geq 0), \\ |\lambda|>1 & (\operatorname{Re} \lambda<0) .\end{cases}
$$

The torus $\mathbb{C} / \Lambda_{i}$ is called a square torus. The torus $\mathbb{C} / \Lambda_{e^{\pi i / 3}}$ is called a hexagonal torus. The projection $\psi_{\lambda}: \mathbb{C} \rightarrow \mathbb{C} / \Lambda_{\lambda}$ is the universal covering. We define $\tilde{a}:[0,1] \rightarrow \mathbb{C}$ and $\tilde{b}:[0,1] \rightarrow \mathbb{C}$ by $\tilde{a}(t):=t$ and $\tilde{b}(t):=\lambda t$ respectively. Then $a:=\psi_{\lambda} \circ \tilde{a}$ and $b:=\psi_{\lambda} \circ \tilde{b}$ are closed curves in $\mathbb{C} / \Lambda_{\lambda}$ subject to the relation $a b a^{-1} b^{-1}=1$. The fundamental group $\pi_{1}\left(\mathbb{C} / \Lambda_{\lambda}, \psi_{\lambda}(0)\right)$ is generated by $[a]$ and $[b]$.

A map $\tau: \mathbb{C} / \Lambda_{\lambda} \rightarrow \mathbb{C} / \Lambda_{\lambda}$ is a holomorphic automorphism such that $\tau^{2}$ is the identity map, if and only if $\left(\tau \circ \psi_{\lambda}\right)(z)=\psi_{\lambda}( \pm z)$. There exists a holomorphic automorphism $\tau$ such that $\tau^{2}$ is not the identity map, if and only if $\mathbb{C} / \Lambda_{\lambda}$ is a square torus or a hexagonal torus. In fact, we define $\tilde{\tau}_{m, n}: \mathbb{C} \rightarrow \mathbb{C}$ by

$$
\tilde{\tau}_{m, n}(z)=e^{2 \pi m i / n} z(n=4,6, m=0,1, \ldots, n-1) .
$$

Then $\tau_{m, 6}: \mathbb{C} / \Lambda_{e^{\pi i / 3}} \rightarrow \mathbb{C} / \Lambda_{e^{\pi i / 3}}$ is defined by $\tau_{m, 6} \circ \psi_{e^{\pi i / 3}}:=\psi_{e^{\pi i / 3}} \circ \tilde{\tau}_{m, 6}$. A map $\tau_{m, 6}$ is a holomorphic automorphism of a hexagonal torus. Similarly, $\tau_{m, 4}: \mathbb{C} / \Lambda_{i} \rightarrow \mathbb{C} / \Lambda_{i}$ is defined by $\tau_{m, 4} \circ \psi_{i}:=\psi_{i} \circ \tilde{\tau}_{m, 4}$. A map $\tau_{m, 4}$ is a holomorphic automorphism of a square torus.

Corollary 4.1. Let $M$ be a square torus and $\omega$ a one-form on $M$ with values in $\mathbb{R}^{n} \subset C \ell_{n}$, satisfying $\omega \wedge \tau_{1,4}{ }^{*} \bar{\omega}-\tau_{1,4}{ }^{*} \omega \wedge \bar{\omega} \neq 0$. A one-form $\omega$ is exact, if and only if $\omega$ is closed and

$$
\Phi\left(\left(\llbracket \omega \rrbracket, \llbracket \tau_{1,4}{ }^{*} \omega \rrbracket\right)-\left(\llbracket \tau_{1,4}{ }^{*} \omega \rrbracket, \llbracket \omega \rrbracket\right)\right)=0 .
$$

Proof. Let $M$ be a square torus. We see that

$$
h\left(\tau_{1,4}\right)=\left(\begin{array}{cc}
0 & -1 \\
1 & 0
\end{array}\right) .
$$

Let $m=1, r_{1}=1$, and $\mu_{1}=\tau_{1,4}$. Then

$$
\begin{gathered}
J_{2} h\left(\tau_{1,4}\right)^{T}-h\left(\tau_{1,4}\right) J_{2}=\left(\begin{array}{cc}
-2 & 0 \\
0 & -2
\end{array}\right)=B\left(\tau_{1,4}\right), \\
C\left(\tau_{1,4}\right)=\tilde{C}\left(\tau_{1,4}\right)=0
\end{gathered}
$$




$$
B\left(\tau_{1,4}\right)+\tilde{C}\left(\tau_{1,4}\right)=\left(\begin{array}{cc}
-2 & 0 \\
0 & -2
\end{array}\right) .
$$

Then Corollary 4.1 holds by Theorem 1.1.

Corollary 4.2. Let $M$ be a hexagonal torus and $\omega$ a one-form on $M$ with values in $\mathbb{R}^{n} \subset C \ell_{n}$, satisfying $\omega \wedge \tau_{1,6}{ }^{*} \bar{\omega}-\tau_{1,6}{ }^{*} \omega \wedge \bar{\omega} \neq 0$. A one-form $\omega$ is exact, if and only if

$$
\Phi\left(\left(\llbracket \omega \rrbracket, \llbracket \tau_{1,6}{ }^{*} \omega \rrbracket\right)-\left(\llbracket \tau_{1,6}{ }^{*} \omega \rrbracket, \llbracket \omega \rrbracket\right)\right)=0 .
$$

Proof. Let $M$ be a hexagonal torus. We see that

$$
h\left(\tau_{1,6}\right)=\left(\begin{array}{cc}
0 & -1 \\
1 & 1
\end{array}\right) .
$$

Let $m=1, r_{1}=1$, and $\mu_{1}=\tau_{1,6}$. Then

$$
\begin{gathered}
J_{2} h\left(\tau_{1,6}\right)^{T}-h\left(\tau_{1,6}\right) J_{2}=\left(\begin{array}{cc}
-2 & 1 \\
1 & -2
\end{array}\right), \\
B\left(\tau_{1,6}\right)=\left(\begin{array}{cc}
-2 & 0 \\
0 & -2
\end{array}\right), C\left(\tau_{1,6}\right)=\left(\begin{array}{cc}
0 & 1 \\
1 & 0
\end{array}\right), \tilde{C}\left(\tau_{1,6}\right)=\left(\begin{array}{ll}
0 & 1 \\
1 & 0
\end{array}\right), \\
B\left(\tau_{1,6}\right)+\tilde{C}\left(\tau_{1,6}\right)=\left(\begin{array}{cc}
-2 & 1 \\
1 & -2
\end{array}\right) .
\end{gathered}
$$

Then Corollary 4.2 holds by Theorem 1.1.

We collect statements for quaternionic-valued one-forms, which are obtained in a similar fashion as above. We omit their proof.

Corollary 4.3. Let $M$ be a square torus and $\omega$ a one-form on $M$ with values in $\mathbb{R}^{4} \cong \mathbb{H}$, satisfying $\omega \wedge \tau_{1,4}{ }^{*} \bar{\omega}-\tau_{1,4}{ }^{*} \omega \wedge \bar{\omega} \neq 0$. A one-form $\omega$ is exact, if and only if $\omega$ is closed and

$$
\left(\llbracket \omega \rrbracket, \llbracket \tau_{1,4}{ }^{*} \bar{\omega} \rrbracket\right)-\left(\llbracket \tau_{1,4}{ }^{*} \omega \rrbracket, \llbracket \bar{\omega} \rrbracket\right)=0 .
$$

Corollary 4.4. Let $M$ be a hexagonal torus and $\omega$ a one-form on $M$ with values in $\mathbb{R}^{4} \cong \mathbb{H}$, satisfying $\omega \wedge \tau_{1,6}{ }^{*} \bar{\omega}-\tau_{1,6}{ }^{*} \omega \wedge \bar{\omega} \neq 0$. A one-form $\omega$ is exact, if and only if

$$
\left(\llbracket \omega \rrbracket, \llbracket \tau_{1,6}{ }^{*} \bar{\omega} \rrbracket\right)-\left(\llbracket \tau_{1,6}{ }^{*} \omega \rrbracket, \llbracket \bar{\omega} \rrbracket\right)=0 .
$$

\section{One-forms on a hyperelliptic curve}

We review a hyperelliptic curve and its automorphisms, and prove Corollary 5.1 .

Let $M$ be the hyperelliptic curve of genus $g$ with affine model (5.1) and $\tau$ be a holomorphic automorphism of $M$ defined by (5.2). If $g=1$, then $M$ is a square torus and $\tau=\tau_{1,4}$.

We label two copies of a sphere, which is identified with $\Sigma=\mathbb{C} \cup$ $\{\infty\}$, sheet I and sheet II. On each sheet, we draw a smooth curve, joining $e^{(2 k-1) \pi i /(g+1)}$ and $e^{2 k \pi i /(g+1)}(k=1, \ldots, g+1)$. These curves are called cuts. We assume that these cuts do not intersect each other. Each cut has 
two banks, called the N-bank and the S-bank. The surface $M$ is constructed by joining every S-bank on sheet I to an N-bank of the corresponding cut on sheet II, and joining every N-bank on sheet I to an S-bank of the corresponding cut on sheet II. We draw a simple closed curve $a_{k}$, winding counterclockwise once, around the cuts joining $e^{(2 k-1) \pi i /(g+1)}$ and $e^{2 k \pi i /(g+1)}$ on sheet I $(k=1, \ldots, g)$. We choose a curve $b_{k}$ starting from a point on the cut from $e^{(2 g+1) \pi i /(g+1)}$ to 1 , going on sheet I, to a point on the cut from $e^{(2 k-1) \pi i /(g+1)}$ to $e^{2 k \pi i /(g+1)}$, and returning on the sheet $\mathbb{I}(k=1, \ldots, g)$. A map $\tau: M \rightarrow M$ defined by $\tau(w, z)=\left(-w, e^{\pi i /(g+1)} z\right)$ is a holomorphic automorphism of $M$. We have a situation similar to a square torus, when a Riemann surface is hyperelliptic. Let $M$ be a hyperelliptic curve of genus $g$ with affine plane model

$$
\left\{(z, w) \in \mathbb{C} \times \mathbb{C} \mid w^{2}=z^{2(g+1)}-1\right\} .
$$

A holomorphic automorphism $\tau: M \rightarrow M$ is defined by

$$
\tau(z, w):=\left(e^{\pi i /(g+1)} z,-w\right) .
$$

We have

$$
\begin{gathered}
h(\tau)=\left(\begin{array}{cc}
O & Q(\tau) \\
R(\tau) & O
\end{array}\right), \\
Q(\tau)=\left(\begin{array}{cccc}
-1 & \ldots \ldots & -1 \\
0 & -1 & \ldots & -1 \\
\vdots & \ddots & \ddots & \vdots \\
0 & \ldots & 0 & -1
\end{array}\right) \\
R(\tau)=\left(\begin{array}{cccccc}
1 & 0 & \ldots \ldots \ldots & \ldots & 0 \\
-1 & 1 & 0 & \ldots \ldots & 0 \\
0 & -1 & 1 & 0 & \ldots & 0 \\
\vdots & \ddots & \ddots & \ddots & \ddots & \vdots \\
0 & \ldots & 0 & -1 & 1 & 0 \\
0 & \ldots \ldots & 0 & -1 & 1
\end{array}\right) .
\end{gathered}
$$

Corollary 5.1. Let $M$ be a hyperelliptic curve of genus two with affine model (5.1), and $\tau$ a holomorphic automorphism defined by (5.2). Let $\omega$ be a oneform with values in $\mathbb{R}^{n} \subset C \ell_{n}$, satisfying $\omega \wedge \tau^{*} \omega-\tau^{*} \omega \wedge \omega \neq 0$. A one-form $\omega$ is exact, if and only if $\omega$ is closed and

$$
\Phi\left(\left(\llbracket \omega \rrbracket, \llbracket \tau^{*} \omega \rrbracket\right)-\left(\llbracket \tau^{*} \omega \rrbracket, \llbracket \omega \rrbracket\right)\right)=0 .
$$

Proof. We have

$$
h(\tau)=\left(\begin{array}{cccc}
0 & 0 & -1 & -1 \\
0 & 0 & 0 & -1 \\
1 & 0 & 0 & 0 \\
-1 & 1 & 0 & 0
\end{array}\right)
$$


Let $m=1, r_{1}=1$, and $\mu_{1}=\tau_{1,6}$. Then

$$
\begin{gathered}
J_{4} h(\tau)^{T}-h(\tau) J_{4}=\left(\begin{array}{cccc}
-2 & -1 & 0 & 0 \\
-1 & -2 & 0 & 0 \\
0 & 0 & -2 & 1 \\
0 & 0 & 1 & -2
\end{array}\right), \\
B(\tau)=\left(\begin{array}{cccc}
-2 & 0 & 0 & 0 \\
0 & -2 & 0 & 0 \\
0 & 0 & -2 & 0 \\
0 & 0 & 0 & -2
\end{array}\right), C(\tau)=\left(\begin{array}{cccc}
0 & -1 & 0 & 0 \\
-1 & 0 & 0 & 0 \\
0 & 0 & 0 & 1 \\
0 & 0 & 1 & 0
\end{array}\right) \\
\tilde{C}(\tau)=\left(\begin{array}{cccc}
0 & 1 & 0 & 0 \\
1 & 0 & 0 & 0 \\
0 & 0 & 0 & 1 \\
0 & 0 & 1 & 0
\end{array}\right), \\
\tilde{C}(\tau)= \\
\left.\begin{array}{cccc}
-2 & 1 & 0 & 0 \\
1 & -2 & 0 & 0 \\
0 & 0 & -2 & 1 \\
0 & 0 & 1 & -2
\end{array}\right) .
\end{gathered}
$$

Then Corollary 4.2 holds by Theorem 1.1.

As in the previous section, we have a similar statement as above for quaternionic-valued one-forms. We omit the proof.

Corollary 5.2. Let $M$ be a hyperelliptic curve of genus two with affine model (5.1), and $\tau$ a holomorphic automorphism defined by (5.2). Let $\omega$ be a oneform with values in $\mathbb{R}^{4} \cong \mathbb{H}$, satisfying $\omega \wedge \tau^{*} \bar{\omega}-\tau^{*} \omega \wedge \bar{\omega} \neq 0$. A one-form $\omega$ is exact, if and only if $\omega$ is closed and

$$
\left(\llbracket \omega \rrbracket, \llbracket \tau^{*} \bar{\omega} \rrbracket\right)-\left(\llbracket \tau^{*} \omega \rrbracket, \llbracket \bar{\omega} \rrbracket\right)=0 .
$$

\section{Surfaces in Euclidean four-space}

We prove Corollary 1.4 and Corollary 1.5.

Firstly, we review the theory of surfaces in terms of quaternions (see [2]). Let $f: M \rightarrow \mathbb{H}$ be a surface. Then there exists $N: M \rightarrow S^{2} \subset \operatorname{Im} \mathbb{H}$ such that $* d f=N d f$. Let $\mathcal{H}: M \rightarrow \mathbb{H}$ be the mean curvature vector of $f$. Then the following equation holds.

$$
-2 d f \overline{\mathcal{H}}=* d N+N d N .
$$

Differentiating this equation, we have

$$
2 d f \wedge d \overline{\mathcal{H}}=d * d N+d N \wedge d N
$$

Proof of Corollary 1.4. We assume that $N, \mathcal{H}$, and $\omega$ satisfy the assumption. Then

$$
2 d \omega \overline{\mathcal{H}}=2 d(\omega \overline{\mathcal{H}})+2 \omega \wedge d \overline{\mathcal{H}}=0
$$


Hence $\omega$ is a closed one-form. By Theorem 1.1, the one-form $\omega$ is exact. Then there exists a map $f: M \rightarrow \mathbb{H}$ with $d f=\omega$. By the assumption for $\omega$, we have $* d f=N d f$. Hence, $f$ is a surface with $* d f=N d f$ and mean curvature vector field $\mathcal{H}$.

The following is the case where a surface takes values in $\mathbb{R}^{3} \cong \operatorname{Im} \mathbb{H}$.

Corollary 6.1. We assume that there exist $\mu_{1}, \ldots, \mu_{m} \in \mathcal{A}$ and $r_{1}, \ldots, r_{m} \in$ $\mathbb{R} \backslash\{0\}$, satisfying one of the following conditions:

1. $\sum_{l=1}^{m}\left(r_{l} B\left(\mu_{l}\right)-\left|r_{l}\right| \tilde{C}\left(\mu_{l}\right)\right)$ is positive definite,

2. $\sum_{l=1}^{m}\left(r_{l} B\left(\mu_{l}\right)+\left|r_{l}\right| \tilde{C}\left(\mu_{l}\right)\right)$ is negative definite.

We assume that maps $N: M \rightarrow S^{2} \subset \operatorname{Im} \mathbb{H}$ and $H: M \rightarrow \mathbb{R}$, and a non-zero one-form $\omega$ on $M$ satisfy

$$
\begin{gathered}
2 \omega H=N * d N-d N,-2 \omega \wedge(d H N+H d N)=d * d N+d N \wedge d N, \\
\sum_{l=1}^{m} r_{l}\left(\omega \wedge \mu_{l}{ }^{*} \bar{\omega}-\mu_{l}{ }^{*} \omega \wedge \bar{\omega}\right) \neq 0, \\
\sum_{l=1}^{m} r_{l}\left[\left(\llbracket \omega \rrbracket, \llbracket \mu_{l}{ }^{*} \bar{\omega} \rrbracket\right)-\left(\llbracket \mu_{l}{ }^{*} \omega \rrbracket, \llbracket \bar{\omega} \rrbracket\right)\right]=0 .
\end{gathered}
$$

Then there exists a surface $f: M \rightarrow \operatorname{Im} \mathbb{H} \cong \mathbb{R}^{3}$ with mean curvature $H$, such that $d f=\omega$ and $* d f=N d f=-d f N$.

Proof. For a surface $f: M \rightarrow \operatorname{Im} \mathbb{H}$, we have $* d f=N d f=-d f N$ with Gauss map $N: M \rightarrow S^{2} \subset \operatorname{Im} \mathbb{H}$. The mean curvature vector of $f$ is $\mathcal{H}=H N$ with mean curvature function $H$. Then this corollary follows from Corollary 1.4.

Proof of Corollary 1.5. A harmonic map $N: M \rightarrow S^{2} \subset \operatorname{Im} \mathbb{H}$ satisfies the equation

$$
d * d N=N d N \wedge * d N .
$$

Let $2 \omega H=N * d N-d N(H \in \mathbb{R} \backslash\{0\})$. Then

$$
\begin{gathered}
-2 \omega \wedge(d H N+H d N)=-(N * d N-d N) \wedge d N \\
=-N * d N \wedge d N+d N \wedge d N=d * d N+d N \wedge d N .
\end{gathered}
$$

Then Corollary 1.5 follows from Corollary 6.1 .

\section{References}

[1] R. L. Bryant, Conformal and minimal immersions of compact surfaces into the 4-sphere. J. Differential Geom. 17 (1982), 455-473.

[2] F. E. Burstall, D. Ferus, K. Leschke, F. Pedit and U. Pinkall, Conformal geometry of surfaces in $S^{4}$ and quaternions. Lecture Notes in Mathematics 1772. Springer-Verlag, 2002. 
[3] H. M. Farkas and I. Kra, Riemann surfaces. 2nd ed. Graduate Texts in Mathematics 71. Springer-Verlag, 1992.

[4] Th. Friedrich, On the spinor representation of surfaces in Euclidean 3-space. J. Geom. Phys. 28 (1998), 143-157.

[5] Th. Friedrich, On superminimal surfaces. Arch. Math. (Brno) 33 (1997), 41-56.

[6] D. A. Hoffman and R. Osserman, The Gauss map of surfaces in $\mathbf{R}^{n}$. J. Differential Geom. 18 (1983), 733-754.

[7] G. Kamberov, P. Norman, F. Pedit, and U. Pinkall, Quaternions, spinors, and surfaces. Contemporary Mathematics 299. American Mathematical Society, 2002.

[8] K. Kenmotsu, Weierstrass formula for surfaces of prescribed mean curvature. Math. Ann. 245 (1979), 89-99.

[9] K. Kenmotsu, Surfaces with constant mean curvature. Translations of Mathematical Monographs 221. American Mathematical Society, 2003.

[10] B. G. Konopelchenko and G. Landolfi, Induced surfaces and their integrable dynamics. II. Generalized Weierstrass representations in 4-D spaces and deformations via DS hierarchy. Stud. Appl. Math. 104 (2000), 129-169.

[11] B. G. Konopelchenko and G. Landolfi, Generalized Weierstrass representation for surfaces in multi-dimensional Riemann spaces. J. Geom. Phys. 29 (1999), 319-333.

[12] B. G. Konopelchenko, Induced surfaces and their integrable dynamics. Stud. Appl. Math. 96 (1996), 9-51.

[13] R. Osserman, A survey of minimal surfaces. 2nd ed. Dover Publications Inc., 1986.

[14] F. Pedit and U. Pinkall, Quaternionic analysis on Riemann surfaces and differential geometry. Proceedings of the International Congress of Mathematicians, Vol. II (Berlin, 1998). Doc. Math. Extra Vol. II, 389-400 (electronic) (1998)

[15] I. A. Taimanov, The Weierstrass representation of spheres in $\mathbf{R}^{3}$, Willmore numbers, and soliton spheres. Proc. Steklov Inst. Math. 225 (1999), 322-343.

[16] I. A. Taimanov, The Weierstrass representation of closed surfaces in $\mathbf{R}^{3}$. Funct. Anal. Appl. 32 (1998), 258-267.

[17] I. A. Taimanov, Surfaces of revolution in terms of solitons. Ann. Global Anal. Geom. 15 (1997), 419-435.

[18] I. A. Taimanov, Modified Novikov-Veselov equation and differential geometry of surfaces. Solitons, geometry, and topology: on the crossroad, 133-151, Amer. Math. Soc. Transl. Ser. 2, 179, Amer. Math. Soc., 1997.

Katsuhiro Moriya

Institute of Mathematics

University of Tsukuba

1-1-1 Tennodai

Tsukuba-shi

Ibaraki-ken 305-8571

Japan

e-mail: moriya@math.tsukuba.ac.jp 